\title{
Clinical application of the activity index to parameter for evaluation of electromyographic activity of the masticatory muscles
}

\author{
Takashi Tanaka \\ Department of Prosthetic Dentistry, Programs for Applied Biomedicine Division of Cervico-Gnathostomatology, Hiroshima University Graduate School \\ of Biomedical Sciences, Hiroshima, Japan.
}

\begin{abstract}
The purpose of this study was to evaluate the relationship between the intended direction of clenching and changes in the applied activity index of the masticatory muscles. The subjects consisted of twelve male volunteers (average age of 26.3 years). The surface electromyographic activities of the anterior and posterior parts of the temporal muscles, the deep posterior part of the masseter muscle and the superficial central part of the masseter muscle were recorded during the intended clenching in vertical, anterior and posterior directions. The changes of the applied activity index (the relative different value between the examined muscle activity and the superficial central part of the masseter muscle activity) were evaluated. The applied activity indexes of the anterior and posterior parts of the temporal muscles and the deep posterior part of the masseter muscle decreased significantly during the intended clenching in the posterior direction. Those of the anterior and posterior parts of the temporal muscles increased significantly during the intended clenching in the anterior direction. Each applied activity index changed corresponding to the differences of the running directions in the sagittal plane between the superficial masseter muscle and these three muscles. The applied activity indexes of the anterior and posterior parts of the temporal muscles and the deep posterior part of the masseter muscle significantly changed during clenching in anteroposterior direction. Therefore, it was suggested that the applied activity indexes of these three muscles could be used as a parameter to indicate the anteroposterior direction of force on the lower jaw.
\end{abstract}

Key words: electromyographic, activity index, direction, clenching

Correspondence: Hitoshi Abekura, Department of Prosthetic Dentistry, Programs for Applied Biomedicine Division of CervicoGnathostomatology, Hiroshima University Graduate School of Biomedical Sciences, 1-2-3 Kasumi, Minami-ku, Hiroshima, 734-8553, Japan. Tel 0081-82-257-5682, Fax 0081-82-257-5684, E-mail: abekura@ hiroshima-u.ac.jp

\section{INTRODUCTION}

The temporo mandibular joint (TMJ) has an anterior articular disk and is rich in blood vessels and nerves posteriorly. ${ }^{1}$ Therefore, it is thought that force imparted on the posterior tissue of TMJ by the mandibular condyle has an adverse.effect. Therefore, the evaluation of the quantity and also the direction of force imparted around TMJ are clinically important. Previous studies have examined the tension applied to the TMJ from such a viewpoint. 2,3

Some studies reported a strong relationship between the direction of the clenching force and the ratio of masticatory muscle activities. ${ }^{4-7}$ Therefore, a change in the ratio of muscle activities is considered to be able to show a change in the direction of the clenching force, and could therefore become a meaningful parameter. The activity index advocated by Naeije et al. ${ }^{8}$ was often adopted as parameters to objectively show the ratio of muscle activities. These parameters can overcome the influences of different conditions, such as variations in the thickness of the subcutaneous fat or electric resistance between electrodes and so on, which varies from patient to patient when the electromyography (EMG) is used. Naeije ${ }^{8}$ and Visser et $a l^{9}$ insisted that these influences can be excluded by converting muscle activities to an index.
Surface electromyograms are clinically easy to record, and both sides of the masseter muscle and anterior parts of temporal muscles are often used as electrode attachment sites. These muscle bundles are broad, and their functions vary. ${ }^{10-12}$ There are other areas which can also be measured comparatively easily by the surface electromyogram, ${ }^{4,13}$ and it may also be useful to take electromyograms from each part. The ratio of muscle activities relates to the direction of the clenching force because the clenching force results from multiple muscle activities.

The purpose of this study was to evaluate the relationship between the intended direction of clenching and changes in the applied activity index (parameter: the relative different value between the examined muscle activity and the superficial central part of the masseter muscle activity) for the anterior and posterior parts of the temporal muscles, and the deep posterior parts of the masseter muscles.

\section{MATERIALS AND METHODS}

The subjects consisted of twelve male volunteers (average of 26.3 years with a standard deviation of 5.4 years) had normal occlusion, i.e. nearly a class 1 molar relation, and were without clinical evidence of symptoms of Temporo Mandibular Disorder (TMD) or unusually 
strong maxillofacial forms. None of the subjects had tooth defects excluding the presence or absence of wisdom teeth, dental caries and severe or moderate periodontal disease. Each subject was informed about the aim and procedures of this study, and gave their informed consent prior to the start of the study.

The EMG activities were amplified and converted from analog to digital with a sample frequency of $2000 \mathrm{~Hz}$, and recorded on the hard disk of a personal computer with the BIOPAC research system (MP100 WS BIOPAC Systems, BIOPAC Systems Inc., Santa Barbara, USA ). The EMG activities were recorded by attaching bipolar silver-silver chloride electrodes (EL204S, BIOPAC Systems Inc., Santa Barbara, USA) in parallel to the direction of the muscle fiber, and ground electrodes were attached to the central part of the frontal head area. When it was necessary to control the clenching level, subjects were able to control their EMG level through visual feedback looking at an oscilloscope displaying the summated rectified EMG signal of central part of masseter muscle.

Several parts of the muscles were selected, i.e. the left anterior part of the temporal muscle, the left posterior part of the temporal muscle, the left superficial central part of the masseter muscle and the left deep posterior part of the masseter muscle (Figure 1). For the anterior part of the temporal muscle, the mid-point between the electrodes was placed $40 \mathrm{~mm}$ ahead of the anterior border of the outer ear canal and $40 \mathrm{~mm}$ above the Frankfort plane. For the posterior part of the temporal muscle, a place superior to the auricle and outer ear canal was decided for one of the bipolar electrodes and a place $15 \mathrm{~mm}$ behind that, for another bipolar electrode. Electrodes were attached to these places along the general direction of the muscle fiber, after vigorous skin cleaning. The muscle bundle of the superficial part of the masseter muscle was confirmed by palpation. Surface electrodes were placed $15 \mathrm{~mm}$ apart on the skin surface, in line with the general direction of the muscle fibers. The mid-point between the electrodes was located on the center of this muscle for the central part of the masseter muscle.The place for the left deep posterior part of the masseter muscle was decided as follows: Part of the zygomatic arch, whose inferior border was confirmed by palpation, was decided on as the upper place to attach one of the bipolar electrodes. A place $15 \mathrm{~mm}$ downward of the upper electrode, reported as the area to palpate the deep part of the masseter muscle for examining TMD, was decided on as the lower place to attach another electrode.

Firstly, the influence of the clenching level on the applied activity index was examined. Subjects were

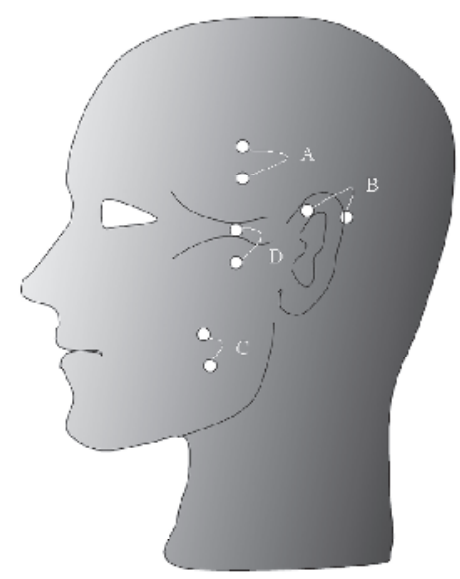

Figure 1. Location of electrodes.

A: anterior part of temporal muscle, B: posterior part of temporal muscle, C: superficial central part of masseter muscle, D: deep posterior part of masseter muscle

asked to clench at $25,50,75$ and $100 \%$ of their maximal voluntary clenching level (MVC) for 5 seconds at the intercuspal position (ICP) through a visual feedback. Next, the influence of the intended clenching direction on the applied activity index was examined. EMG activities were recorded during the intended clenching in vertical, anterior and posterior directions at the intercuspal position. Subjects were asked to clench as strongly as possible and toward the intended direction as constantly as possible. Each clenching was maintained for 3 seconds while measurements were performed, and this was carried out twice. At least one minute rest period was given between each clenching to avoid muscle fatigue.

The whole sampled EMG data were filtered through the range between 50 and $1000 \mathrm{~Hz}$ and rectified. From each of the recorded EMG activities, stable EMG activities were selected respectively, and the integrated EMG activities were calculated. Finally, the four integrated EMG activities in each clenching condition were averaged and used to calculate the next parameter. To evaluate the change of each muscle activity, the activity index, advocated by Naeije, McCarroll and Weijes ${ }^{8}$ was used and applied as Figure 2 for present study.

Paired Student's t-tests were performed to determine difference between each condition. A Bonferroni correction of the alpha-level for statistical analysis was performed to avoid type I error increased by multiplicity. Statistical analyses were performed with significance set at the 0.05

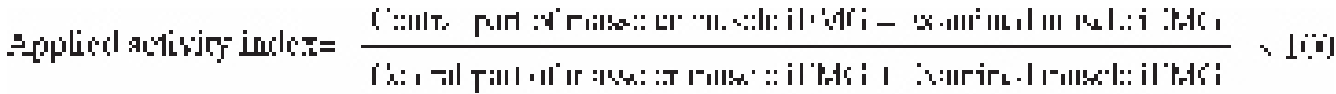

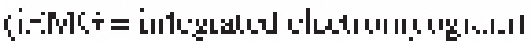

Figure 2. Formula for the applied activity index. ${ }^{8}$ 
probability level.

\section{RESULTS}

The differences in clenching level, i.e. $25 \%, 50 \%, 75 \%$, and $100 \%$ MVC, did not influence the applied activity index for all muscles (Figure 3, 4).

The changes in the applied activity index for the anterior and posterior parts of the temporal muscles showed similar results to each other. The applied activity index for these muscles in the vertical direction was significantly lower than in the anterior direction, and the index in the posterior direction was significantly lower than in the vertical direction (Figure 5). The applied activity index for the deep posterior part of masseter muscle in the posterior direction was significantly lower than in the vertical and anterior directions (Figure 6).

\section{DISCUSSION}

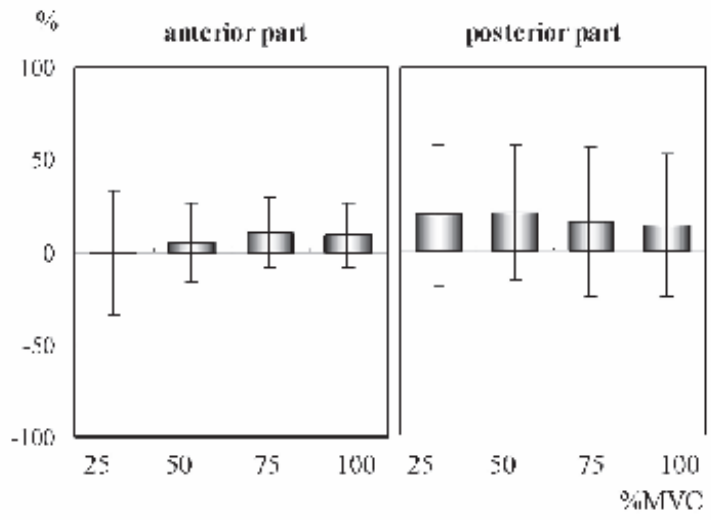

Figure 3. Changes in the applied activity index for the temporal muscles caused by alteration in the clenching level.

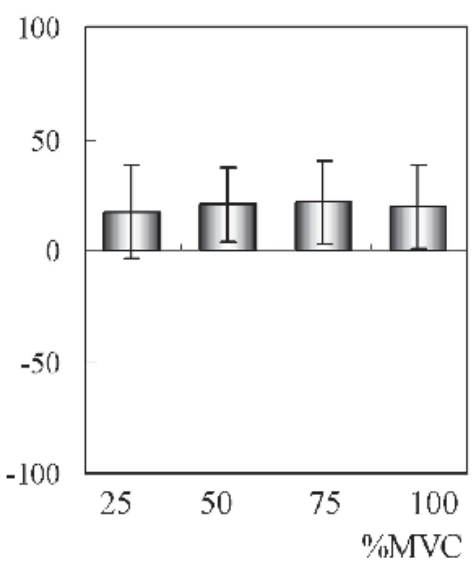

Figure 4. Changes in the applied activity index for deep posterior part of masseter muscle caused by alteration in the clenching level.

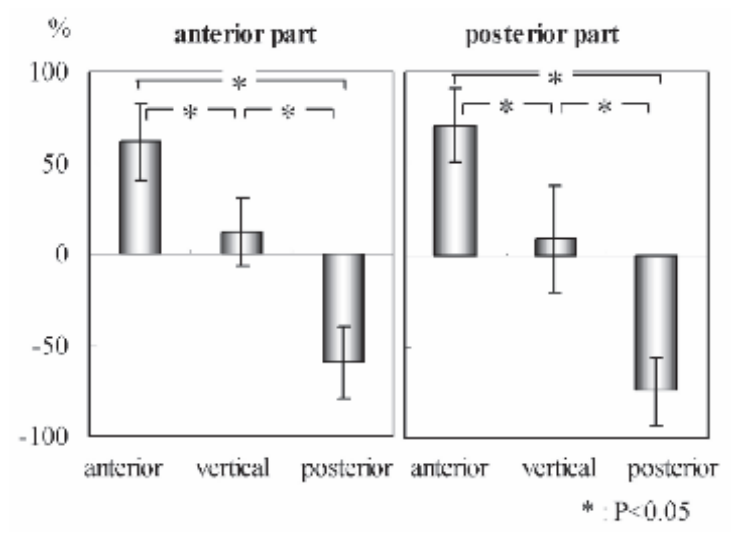

Figure 5. Changes in the applied activity index for the temporal muscles caused by alteration in the clenching direction.

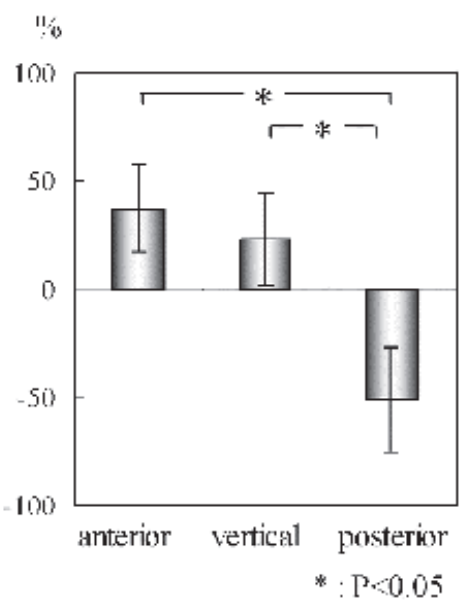

Figure 6. Changes in the applied activity index for the deep posterior part of masseter muscles caused by alterations in clenching direction.

The activity index, one of the proposed parameters, shows the relative value of the activities of the center of the masseter muscle against the activities of the anterior part of the temporal muscle, as an applicable muscle. ${ }^{8}$ The main direction of the clenching force aligns with the running of the masseter muscle fibers, and this muscle is considered to be one of the main working muscles in the generation of clenching force. Other muscle activity increases the power and modifies the direction of the power generated by the masseter muscle. According to the formula of the applied activity index, this parameter is calculated by dividing the difference in the values between the masseter muscle and examining muscle activities by the total value of both muscle activities. The total value is in proportion to the clenching strength. Therefore this parameter is expected to reflect the direction of the power regardless of the clenching strength.

There are other muscles that modify the center of masseter muscle activity except for the anterior part of the 
temporal muscle, and it is possible that similar parameters to the activity index are available for each muscle. The running of the posterior part of the temporal muscle does not identify that of the anterior part, so it is meaningful to examine both muscle activity patterns because the functions are different from each other. ${ }^{11}$ Not only the running direction of the deep posterior masseter muscle but also origin and insertion are different from the superficial masseter muscle, ${ }^{14}$ and this muscle is also functionally different. ${ }^{13}$ Therefore, to examine this muscle's activity is beneficial. Oppressive pain occurs at the area of the deep posterior masseter muscle in patients with TMD. ${ }^{15}$ This muscle is clinically important for these reasons but a few EMG studies have been conducted on the deep masseter muscle. ${ }^{13,16}$ Most of the deep masseter muscle is covered with superficial masseter muscle, but not the area just anterior to the temporomandibular joint. ${ }^{14}$ Belser ${ }^{13}$ examined the electromyographic activity of this part as a target by means of surface and needle electrodes. A large muscle activity can be recorded during clenching in the posterior direction using both needle and surface electrodes. These four muscles, that is, the superficial central and deep posterior part of the masseter muscles and the anterior and posterior parts of the temporal muscles, are clinically important and easy to attach surface electrodes to. ${ }^{4}$ Therefore, these muscles were selected for the present study.

Because the running of the muscles examined in this study varies in the sagittal plane, the clenching force direction was designated in the sagittal plane, that is, in the anterior and posterior directions. Because the regulation of the clenching level makes it difficult for the subject to perform clenching in the designated direction, each subject was asked to clench in the intended direction at the maximal voluntary level. Before recording EMG during clenching, each subject practiced anterior and posterior mandibular sliding movements so that they could perform the intended direction of clenching at the intercuspal position. Because the clenching level was not regulated during anterior and posterior intended clenching, changes in the applied activity indexes may have been influenced by the intensity of clenching. However, we did not observe significant influence of differences in clenching level intensity on the applied activity index. Therefore, the results of Figure 5 and 6 indicated the influence of the clenching direction.

The power direction of each muscle activity depends on the running of the muscle fibers. When subjects clench in the posterior direction, it is considered that the deep posterior part of the masseter muscle and the anterior and posterior parts of the temporal muscle may become active and the applied activity indexes of these muscles decrease. These muscle activities decrease and the indexes increase when subjects clench in the anterior direction. The running directions of these muscles incline backward in the order of the posterior part of temporal muscle, the anterior part of temporal muscle, the deep posterior part of the masseter muscle, and the superficial central part of the masseter muscle in the sagittal plane. ${ }^{13}$ Because the posterior part of the temporal muscle runs mostly in the posterior direction, this applied activity index changed most noticeably. The change in the numerical value of the deep part of the masseter muscle was similar to the temporal muscle, because the directions of both muscles are aligned with each other.

If the running direction of these examined muscles is taken into consideration, the relationship between the direction of the clenching force and the change in the applied activity index could be explained well. It was suggested that the applied activity indexes of these three muscles, the deep posterior part of the masseter muscle, and the anterior and posterior parts of the temporal muscles, could be used as a parameter to indicate the anteroposterior direction of force on the lower jaw.

\section{REFERENCES}

1. Scapino RP. The posterior attachment: Its structure, function, and appearance in TMJ imaging studies. Part 1. J Craniomandib Disord Facial Oral Pain 1991; 5:83-95.

2. Ferrario VF, Sforza C. Biomechanical model of the human mandible in unilateral clench: distribution of temporomandibular joint reaction forces between working and balancing sides. J Prosthet Dent, 1994; 72:169-76.

3. Osborn JW. Biomechanical implications of lateral pterygoid contribution to biting and jaw opening in humans. Arch Oral Biol 1995; 40:1099-108.

4. Wood WW. A review of masticatory muscle functuion. J Prosthet Dent 1987; 57:222-32.

5. MacDonald JW, Hannam AG. Relationship between occlusal contacts and jaw-closing muscle activity during tooth clenching: Part II. J Prosthet Dent 1984; 52:862-7.

6. Mao J, Osborn JW. Direction of a bite force determines the pattern of activity in jaw-closing muscles. J Dent Res 1994; 73:1112-20.

7. Van Eijden TM. Jaw muscle activity in relation to the direction and point of application of bite force. J Dent Res 1990; 69:901-5.

8. Naeije M, McCarroll RS, Weijes WA. Electromyographic activity of the human masticatory muscles during submaximal clenching in the inter-cuspal position. J Oral Rehabil 1989; 16:63-70.

9. Visser A, McCarroll RS, Naeije M. Masticatory Muscle activity in different jaw relations during submaximal clenching efforts. J Dent Res 1992;71:372-9.

10. McMillan AS, Hannam AG. Task-related behavior of motor units in different regions of the human masseter muscle. Arch Oral Biol 1992; 37:849-57.

11. Blanksma NG, Van Eijden TM. Electromyographic heterogeneity in the human temporalis muscle. J Dent Res 1990; 69:1686-90.

12. Blanksma NG, Van Eijden TM, Weijs WA. Electromyographic heterogeneity in the human masseter muscle. J Dent Res 1992; 71: 47-52.

13. Belser UC, Hannam AG. The contribution of the deep fibers of the masseter muscle to selected tooth-clenching and chewing tasks. J Prosthet Dent 1986; 56:629-35.

14. Eriksson PO, Thornell LE. Histochemical and morphological musclefibre characteristics of the human masseter,the medial pterygoid and the temporal muscles. Archs oral Biol, 1983; 28:781-95.

15. Travell J, Simons DG. Myofascial Pain and Dysfunction: The Trigger Point Manual. Baltimore: Williams and Wilkins, 1983. p. 45-102,

16. Santana U, Mora MJ. Electromyographic analysis of the masticatory muscles of patients after complete rehabilitation of occlusion with protection by non-working side contacts. J Oral Rehabil 1995; 22:57-66. 